\title{
cTrust: Trust Aggregation in Cyclic Mobile Ad Hoc Networks ${ }^{\star}$
}

\author{
Huanyu Zhao, Xin Yang, and Xiaolin Li \\ Scalable Software Systems Laboratory, Department of Computer Science \\ Oklahoma State University, Stillwater, OK 74078, USA \\ \{huanyu, xiny, xiaolin\}@cs.okstate.edu
}

\begin{abstract}
In a Cyclic Mobile Ad Hoc Network (CMANET) where nodes move cyclically, we formulate trust management problems and propose the cTrust scheme to handle trust establishment and aggregation issues. Unlike trust management in conventional peer-to-peer (P2P) systems, trust management in MANETs is based on simple neighbor trust relationships and also location and time dependent. In this paper, we focus on trust management problem in highly mobility environment. We model trust relations as a trust graph in CMANET to enhance accuracy and efficiency of trust establishment among peers. Leveraging a stochastic distributed BellmanFord based algorithm for fast and lightweight aggregation of trust scores, the cTrust scheme is a decentralized and self-configurable trust aggregation scheme. We use the NUS student contact patterns derived from campus schedules as our CMANET communication model. The analysis and simulation results demonstrate the efficiency, accuracy, scalability of the cTrust scheme. With increasing scales of ad hoc networks and complexities of trust topologies, cTrust scales well with marginal overheads.
\end{abstract}

\section{Introduction}

Research in Mobile Ad Hoc Networks (MANETs) has made tremendous progress in fundamental protocols, routing, packet forwarding and data gathering algorithms, and systems. Different from the conventional networks, in MANETs, nodes carry out routing and packet forwarding functions so that nodes act as terminals and routers. The increasing popularity of these infrastructure-free systems with autonomous peers and communication paradigms have made MANETs prone to selfish behaviors and malicious attacks. MANETs are inherently insecure and untrustful. In MANETs, each peer is free to move independently, and will therefore change its connections to other peers frequently, which results in a very high rate of network topology changes. The communication is usually multi-hop, and each node may forward traffic even unrelated to its own use. The transmission power, computational ability and available bandwidth of each node in MANETs is limited. In reality, we notice that a large part of MANETs

\footnotetext{
* The research presented in this paper is supported in part by National Science Foundation (grants CNS-0709329, CCF-0953371, OCI-0904938, CNS-0923238).
} 
peers have cyclic movement traces and can be modeled as Cyclic Mobile Ad Hoc Networks (CMANETs) which is defined as a kind of MANET where nodes' mobilities are cyclic 5 57. In this paper, we focus on the trust management problem in CMANETs. Conventional centralized trust establishment approaches are not suited well for use within CMANETs scenarios. And to our best knowledge, little research work has investigated the trust issues in CMANETs. Trust establishment in CMANETs is still an open and challenging topic. Unlike the P2P trust, CMANETs are based on naive neighbor trust relationships. Trust in CMANETs is also location and time dependent.

We propose a trust aggregation scheme called cTrust for aggregation of distributed trust information in completely decentralized and highly-dynamic CMANET environments. Our contributions in this work are multifold. (1) we model the movement patterns and trust relationships in CMANET as a trust graph. And we model the most trustable path finding process as the Markov Decision Process (MDP). (2) We propose trust transfer function, value iteration function and distributed trust aggregation algorithm to solve the most trustable path finding problem. This algorithm leverages a stochastic markov chain based distributed Bellman-Ford algorithm, which greatly reduces the message overhead. It requires only localized communication between neighbor peers, and it captures a concise snapshot of the whole network from each peer's perspective. (3) We design the evaluation metrics for cTrust. Using random and scale-free trust topologies, we conduct extensive experimental evaluations based on the NUS student real campus movement trace data.

The structure of the rest paper is as follows. Section 2 presents the related works. Section 3 proposes trust model and the stochastic distributed cTrust aggregation algorithm. We present the simulation results to explore the performance of cTrust in section 4. Section [5] concludes the paper.

\section{Related Work}

The EigenTrust scheme proposed by Kamvar et al. presents a method to obtain a global trust value for each peer by calculating the eigen value from a trust ratings matrix [4. Xiong and Liu developed a reputation-based trust framework PeerTrust [1]. Zhou and Hwang etc. proposed the PowerTrust system for DHTbased P2P networks [14]. H. Zhao and X. Li proposed the concept of trust vector and a trust management scheme VectorTrust for aggregation of distributed trust scores [13, and they also proposed a group trust rating aggregation scheme $\mathrm{H}$ Trust using H-Index technique [12].

In the field of MANETs, Sonja Buchegger and Jean-Yves Le Boudec proposed a reputation scheme to detect misbehavior in MANETs [1]. Their scheme is based on a modified Bayesian estimation method. Sonja Bucheggery and his colleague also proposed a self-policing reputation mechanism [2]. The scheme is based on nodes' locally observation, and it leverages second-hand trust information to rate and detect misbehaving nodes. The CORE system adopts a reputation mechanism to achieve nodes cooperation in MANETs [6]. The goal of CORE 
system is to prevent nodes' selfish behavior. [1, 2, and [6] mainly deal with the identification and isolation of misbehaved nodes in MANETs, but the mobility feature of MANETs is not fully addressed in these previous work. Yan Sun et al. considered trust as a measure of uncertainty, and they presented a formal model to represent, model and evaluate trust in MANETs [9]. Ganeriwal, Saurabh et al. extended the trust scheme application scenario to sensor networks and they built a trust framework for sensor networks [3]. Another Ad Hoc trust scheme is [10] where the trust confidence factor was proposed.

\section{3 cTrust Scheme}

\subsection{Trust Graph in CMANETs}

In CMANETs, nodes have short radio range, high mobility, and uncertain connectivity. Two nodes are able to communicate only when they reach each others' transmission range. When two nodes meet at a particular time, they have a contract probability $P(P \in[0,1])$ that they contact or start some transactions.

The cyclic movement trace graph of a CMANET consisting of three nodes is shown in Figure 1, The unit time is set as 10. Each peer $i$ moving cyclically has motion cycle time $C_{i}$. We can tell from the trace that $C_{A}=30$, $C_{B}=30$ and $C_{C}=20$. The system motion cycle time $C_{S}$ is the Least Common Multiple (LCM) of all the peers' motion cycle time in the network, $C_{S}=$ $\operatorname{lcm}\left(C_{A}, C_{B}, C_{C}\right)=60$. Note that, CMANETs movement traces are not required to be following some shapes. The "cyclic" is explained that if two nodes meet at time $T 0$, they have a high probability to meet after every particular time period $T_{P}$. We represent the movement traces in this paper as some shapes to ease the presentation and understanding.

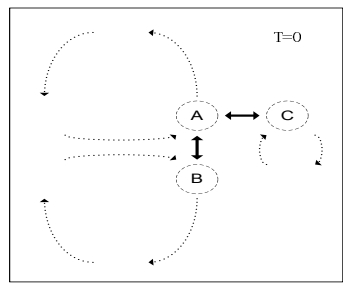

(a) $T=0$

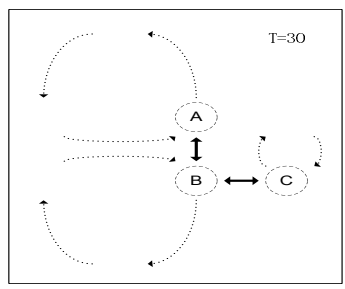

(d) $T=30$

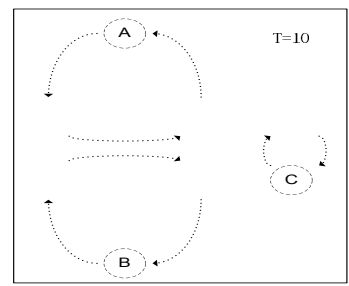

(b) $T=10$

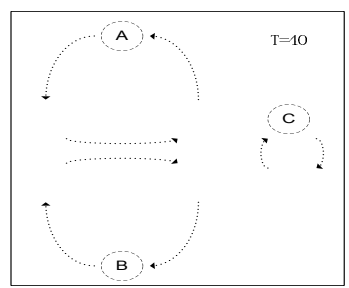

(e) $T=40$

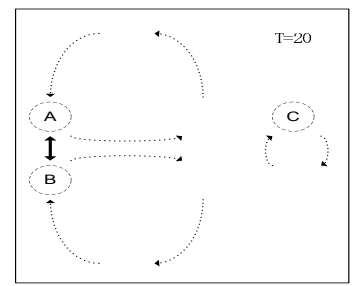

(c) $T=20$

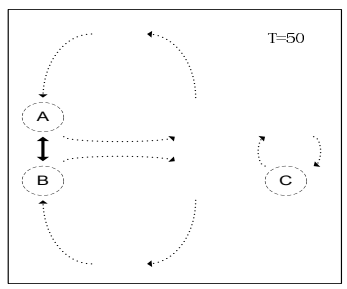

(f) $T=50$

Fig. 1. CMANET Movement Trace Snapshots for One System Cycle $\left(C_{S}=60\right)$ 


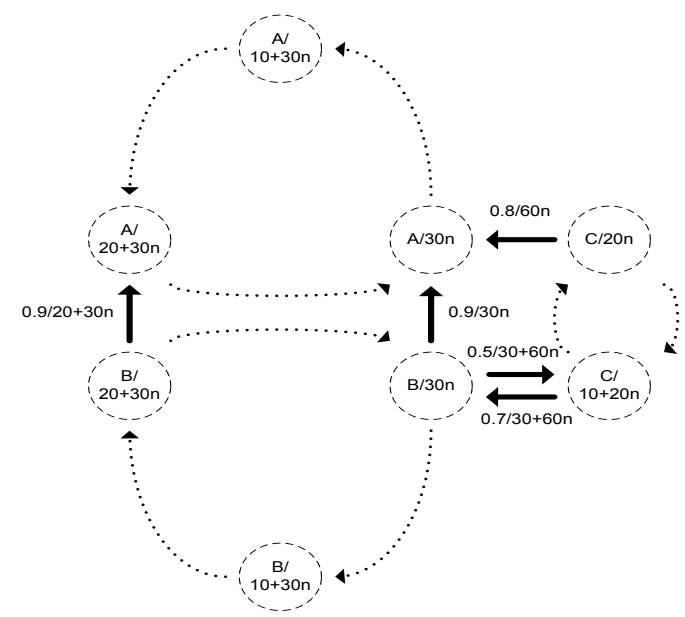

Fig. 2. Trust Graph. The vertices in the graph correspond to peer states (time and location) in a system. An directed solid edge shows the initial trust relation (as trust rating and trust direction) and contact time. The dashed line shows the nodes' movement trace. Each peer maintains a local trust table to store trust information.

To describe the CMANETs system features and trust relationships, we combine the snapshot graph and trust relationships into a directed trust graph as shown in Figure 2, Each node is represented by several states based on periodically appearing locations as the vertices in graph. We represent the states $X_{i}$ as $i / T_{i}\{L o c\}$ where $i$ is the node ID and $T_{i}\{L o c\}$ is appearance time for particular locations. The appearance time is give by,

$$
T_{i}\{L o c\}=T 0_{i}\{L o c\}+C_{i} \times n(n=0,1,2, \ldots)
$$

where $T 0_{i}\{L o c\}$ is the first time node $i$ appears at this location and $C_{i}$ is node $i$ 's motion cycle time. For example, node $A$ appears at three locations. So in trust graph, node $A$ is represented by three states: $A / T_{A}\left\{L o c_{0}\right\}, A / T_{A}\left\{L o c_{1}\right\}$ and $A / T_{A}\left\{L_{o c_{2}}\right\}$. Following Equation (11), we have, $T_{A}\left\{L o c_{0}\right\}=0+C_{A} \times n=30 n$, $T_{A}\left\{L_{o c}\right\}=10+C_{A} \times n=10+30 n, T_{A}\left\{L_{o c}\right\}=20+C_{A} \times n=20+30 n,(n=$ $0,1,2, \ldots)$. The three states generated by node $A$ is $A / 30 n, A /(10+30 n), A /(20+$ $30 n)$. State $X_{i}$ 's one hop direct trust neighbors is represented by the set $H^{\prime}\left(X_{i}\right)$, e.g., $H^{\prime}(B / 30 n)=\{A / 30 n, C /(10+20 n)\}$. The directed dashed lines between states shows nodes' movement trace as state transfer edges in trust graph.

The initial trust relationships are shown by the solid directed edges in the graph. There is an edge directed from peer $i$ to peer $j$ if and only if $i$ has a trust rating on $j$. The value $R_{i, j}\left(R_{i, j} \in[0,1]\right)$ reflects how much $i$ trusts $j$ where $R_{i, j}=0$ indicates $i$ never/distrust trust $j, R_{i, j}=1$ indicates $i$ fully trust $j$. The trust between different states of the same node $i$ is considered as $R_{i, i}=1$.

The trust rating is personalized which means the peer has self-policing trust on other peers, rather than obtaining a global reputation value for each peer. We adopt personalized trust rating because in an open and decentralized CMANET 
environment, peers will not have any centralized infrastructure to maintain a global reputation. The solid trust rating value on the edge could be obtained by applying different functions to consider all the history transactions' importance, date, service quality between two peers. How to rate a service and how to generate and normalize the accurate direct trust ratings are not with the scope of this paper. In this paper, we assume the normalized trust ratings have been generated. What we studied in this paper is the trust aggregation/propagation process in an ad hoc network with high mobility.

Besides trust ratings, each edge is also labeled by a time function showing when two nodes can communicate by this trust link. The appearance time for each link is given by Equation (2):

$$
T_{R_{i, j}}=T 0_{R_{i, j}}+\operatorname{lcm}\left(C_{i}, C_{j}\right) \times n(n=0,1,2, \ldots)
$$

Where $C_{i}, C_{j}$ is the relevant nodes' motion cycle time and $T 0_{R_{i, j}}$ is the first time they meet by this link. The solid edges are represented as $R_{i, j} / T_{R_{i, j}}$. The system trust graph shows all the trust relationships, the moving trace and possible contacts of the network. For example, setting $T=0$ at Figure 2, we obtain the snapshot trace as in Figure 1(a) and the appearing trust links.

\subsection{Trust Path Finding Problems in CMANET}

In cTrust system, each peer maintains a local trust table. The trust table consists of the remote peer ID as entry, the trust rating for each possible remote peer, the next hop to reach the remote peer. Each entry shows only the next hop instead of the whole trust path. Initially, peers's trust tables only contain the trust information of their one hop direct experience.

Due to the communication range and power constrains, peers are not able to communicate with remote peers directly. Suppose peer $i$ wishes to start a transaction with remote peer $k . i$ wishes to infer an indirect trust rating for peer $k$ to check $k$ 's reputation. In cTrust, the trust transfer is defined as follows.

Definition 3.1 (Trust Transfer): If peer $i$ has a trust rating $R_{i, j}$ towards peer $j$, peer $j$ has trust rating $R_{j, k}$ towards peer $k$, then peer $i$ has indirect trust $R_{i, k}=R_{i, j} \otimes R_{j, k}$ towards peer $k$.

In Definition 3.1, $R_{i, j}$ and $R_{j, k}$ can be both direct and indirect trust. Beside the trust rating, peer $i$ also wishes to find a trustable path and depend on the multihop communication to finish this transaction. Among a set of paths between $i$ and $k, i$ tends to choose the Most Trustable Path (MTP).

\section{Definition 3.2 (Most Trustable Path): The most trustable path from peer $i$ to peer $k$ is the trust path yielding highest trust rating $R_{i, k}$.}

MTP is computed as the maximal $\otimes$ production value of all directed edges along a path. And this production will be considered as $i$ 's trust rating towards peer $k$. The MTP provides a trustable communication path, and is used to launch multi-hop transactions with an unfamiliar target peer. cTrust scheme solves the trust rating transfer and MTP finding problems in CMANETs. 


\subsection{Markov Decision Process Model}

Markov Decision Process (MDP) is a discrete time stochastic control process consisting of a set of states. In each state there are several actions to choose. For a state $x$ and an action $a$, the state transition function $P_{x, x^{\prime}}$ determines the transition probabilities to the next state. A reward is also earned for each state transition. We model the MTP finding process as a MDP. We propose value iteration to solve the MTP finding problem.

\section{Theorem 3.1: The MTP finding process is a Markov Decision Process.}

\section{Proof}

Initially, for a sequence of random node states in trust graph $X_{1}, X_{2}, X_{3}, \ldots, X_{t}$, the trust path has the following relation:

$$
\begin{aligned}
\operatorname{Pr}\left(X_{t+1}\right. & \left.=x \mid X_{1}=x_{1}, X_{2}=x_{2} \ldots, X_{t}=x_{t}\right) \\
& =\operatorname{Pr}\left(X_{t+1}=x \mid X_{t}=x_{t}\right)
\end{aligned}
$$

Equation 3 indicates the state transitions of a trust path possess the markov property: the future states depend only on the present state, and are independent of past states.

The components required in a MDP are defined by the following notations:

- $\mathcal{S}$ : state space of $M D P$, the node state set in the trust graph.

- A: action set of MDP, the state transition decisions.

$-P_{x, x^{\prime}}=\operatorname{Pr}\left(X_{t+1}=x^{\prime} \mid X_{t}=x, a_{t}=a\right)$ : the probability that action a in node state $x$ at time $t$ will lead to node $x^{\prime}$ at time $t+1$.

- $R_{x, x^{\prime}}$ : the reward received after transition to state $x^{\prime}$ from state $x$ with transition probability $P_{x, x^{\prime}} . R_{x, x^{\prime}}$ is in terms of trust rating in out scheme.

The state transition probability in state $x$ to state $x^{\prime}$ is computed from normalizing all $x$ 's out trust links (trust ratings).

$$
P_{x, x^{\prime}}=\operatorname{Pr}\left(X_{t+1}=x^{\prime} \mid X_{t}=x\right)=\frac{R_{x, x^{\prime}}}{\sum_{y \in H^{\prime}(x)} R_{x, y}}
$$

In each node state, the next state probability sums to one. The trust path finding process is a stochastic process that all state transitions are probabilistic.

The goal is to maximize the cumulative trust rating for the whole path, typically the expected production from the source peer to the destination peer.

$$
\gamma R_{s_{1}, s_{2}} \otimes \gamma^{2} R_{s_{2}, s_{3}} \otimes \gamma^{3} R_{s_{3}, s_{4}} \otimes \ldots \otimes \gamma^{t} R_{s_{t}, s_{t+1}}
$$

where $\gamma$ is the discount rate and satisfies $0 \leq \gamma \leq 1$. It is typically close to 1 .

Therefore, the MTP finding process is a $\operatorname{MDP}\left(\mathcal{S}, \mathcal{A}, P_{., .}, R_{., .}\right)$.

The solution to this MDP can be expressed as a trust path $\pi$ (MTP), The standard algorithms to calculate the policy $\pi$ is the value iteration process. 


\subsection{Value Iteration}

Section 3.2 presents the trust transfer function $R_{i, k}=R_{i, j} \otimes R_{j, k}$. The upper bound for $R_{i, j} \otimes R_{j, k}$ is $\min \left(R_{i, j}, R_{j, k}\right)$ because the combination of trust cannot exceed any original trust. $R_{i, j} \otimes R_{j, k}$ should be larger than $R_{i, j} \times R_{j, k}$, which avoid a fast trust rating dropping in trust transfer. The discount rate $\gamma(\gamma \in$ $[0,1])$ determines the importance of remote trust information. The trust transfer function $R_{i, j} \otimes R_{j, k}$ needs to meet the following condition:

$$
R_{i, j} \times \gamma R_{j, k} \leq R_{i, j} \otimes R_{j, k} \leq \min \left(R_{i, j}, \gamma R_{j, k}\right)
$$

In cTrust scheme, we set the trust transfer function as:

$$
R_{i, j} \otimes R_{j, k}=\min \left(R_{i, j}, \gamma R_{j, k}\right) \times \sqrt[n]{\max \left(R_{i, j}, \gamma R_{j, k}\right)}
$$

We prove that the given function meets the condition in (6).

\section{Proof}

$$
\begin{gathered}
\max \left(R_{i, j}, \gamma R_{j, k}\right) \leq 1, \text { so, } \min \left(R_{i, j}, \gamma R_{j, k}\right) \times \sqrt[n a]{\max \left(R_{i, j}, \gamma R_{j, k}\right)} \leq \min \left(R_{i, j}, \gamma R_{j, k}\right) \\
\min \left(R_{i, j}, \gamma R_{j, k}\right) \times \sqrt[n_{a}]{\max \left(R_{i, j}, \gamma R_{j, k}\right)} \\
=\frac{\min \left(R_{i, j}, \gamma R_{j, k}\right) \times \max \left(R_{i, j}, \gamma R_{j, k}\right)}{n_{a}}=\frac{R_{i, j} \times \gamma R_{j, k}}{\frac{n_{a}-1}{n_{a}-\sqrt{\max \left(R_{i, j}, \gamma R_{j, k}\right)}}} \geq R_{i, j} \times \gamma R_{j, k}
\end{gathered}
$$

Therefore, we have proved that the trust transfer function (7) meets the condition (6).

By setting up the adjusting factor $n_{a}\left(n_{a}=1,2,3 \ldots\right),\left(R_{i, j} \otimes R_{j, k}\right)$ can be sliding between the upper and lower bound.

In each round of the iteration, the trust table of each node is updated by choosing an action (next hop state in trust graph). The value iteration is executed concurrently for all nodes. It compares the new information with the old trust information and makes a correction to the trust tables based on the new information. The trust tables associated with the nodes are updated iteratively and until they converge. Based on the trust transfer function, the value iteration function is set up as:

$$
R_{i, k}=\max \left(R_{i, k}, \alpha\left[\min \left(R_{i, j}, \gamma R_{j, k}\right) \sqrt[n_{a}]{\max \left(R_{i, j}, \gamma R_{j, k}\right)}\right]\right)
$$

where $R_{i, k}$ is the trust rating towards peer $k$ given peer $i$ 's local trust table, $R_{i, j}$ is the direct link trust and $R_{j, k}$ is the received trust information towards peer $k . \alpha(\alpha \in[0,1])$ is the learning rate. The learning rate determines to what extent the newly acquired trust information will replace the old trust rating. A learning rate $\alpha=0$ indicates that the node does not learn anything, and a learning rate factor $\alpha=1$ indicates that the node fully trusts and learns the new information. At the convergence status of the value iteration, each peer's trust table will contain the trust rating for MTP.

\section{5 cTrust Distributed Trust Aggregation Algorithm}

In the initial stage of an evolving CMANET, pre-set direct trust ratings are stored in local trust tables. However, the direct trust information is limited and 
does not cover all potential interactions. The distributed trust aggregation algorithm gathers trust ratings to any peer in a network (Algorithm 1). In this algorithm, each trust path is aggregated to MTP with highest trust rating towards target peer. Indirect trust information will be added to trust tables and be updated as the aggregation process evolves. The algorithm is implemented based on distributed Bellman-Ford algorithm. Updates are performed periodically where peers retrieve one of their direct trust neighbors' trust tables and replace existing trust ratings with more higher ones in local trust tables, then include relevant neighbors as the next hops.

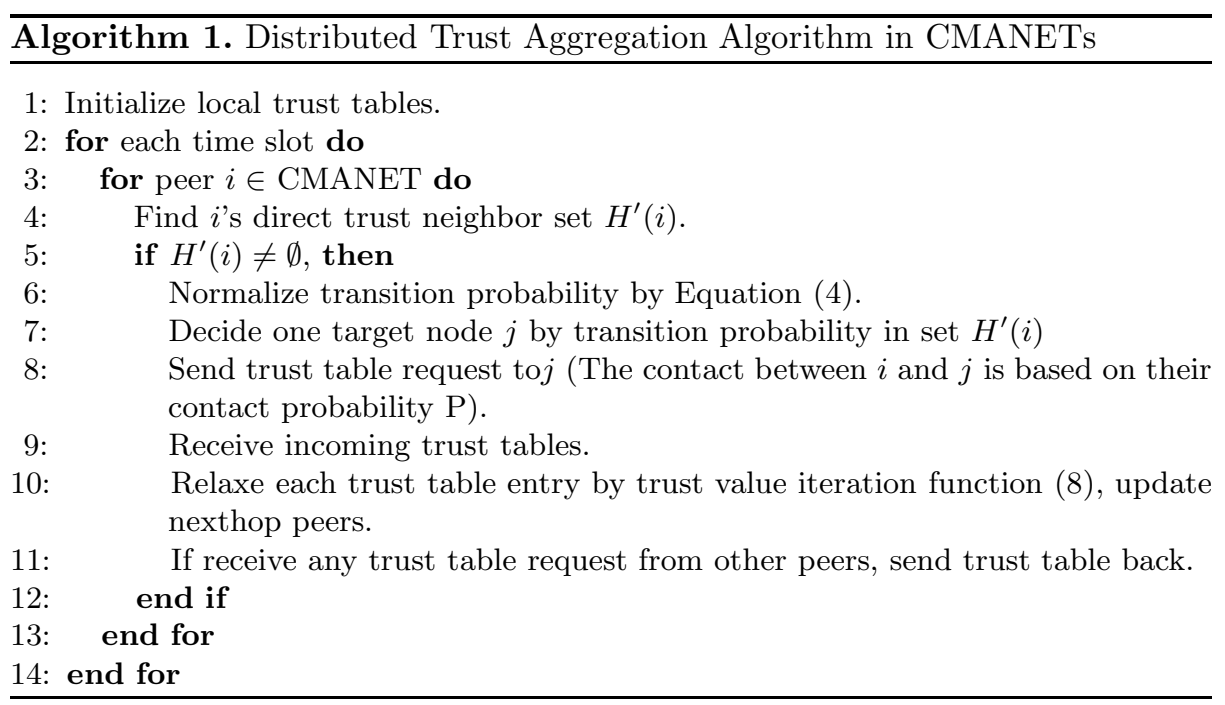

\section{Experimental Evaluation}

\subsection{Experiment Setup}

CMANET Contact Pattern Model. We construct an unstructured network based on the NUS student trace. The data of contact patterns is from the class schedules for the Spring semester of 2006 in National University of Singapore (NUS) among 22341 students with 4875 sessions [78]. For each enrollment student, we have her/his class schedule. It gives us extremely accurate information about the contact patterns among students over large time scales. The contact patterns among students inside classrooms were presented in [7]. Following class schedules, students move around on campus and meet each other when they are in the same session. The trace data set considers only students movements during business hours, and ignores contacts that students hang around campus for various activities outside of class. The time is compressed by removing any idle time slots without any active sessions. So the contacts take place only in classrooms. Two students are within communication range of each other if and 
only if they are in the same classroom at the same time. The sessions can be considered as classes. The unit time is one hour, and a session may last multiple hours. The NUS contact patterns can be modeled as CMANET. In our experiment, 100 to 1000 students are randomly chosen to simulate 100 to 1000 moving peers in CMANET. Following her/his class schedule, each student appears moving cyclically in classrooms. The contact probability $P$ is set as 0.9 which indicates that when two nodes meet, they have a probability of 0.9 to communicate. We considered all 4875 sessions in the data set. The time for the whole system cycle $\left(C_{S}\right)$ is 77 hours (time units).

Trust Topology Model. The random trust topology and scale-free trust topology are used to establish trust relationships in this simulation. In random trust topology, the trust outdegree of a peer follows normal distribution with mean value $\mu_{d}=20,25,30$ and variance $\sigma_{d}^{2}=5$. On the random trust topology, all peers have similar initial trust links. Under the scale-free trust topology, highly active peers possess large numbers of trust links, and most other peers only have small numbers of trust links. The number of trust links follows power law distribution with an scaling exponent $k=2$.

Parameter Setting. The network is configured from 100 nodes to 1000 nodes. The network complexity is represented in terms of nodes' average outdegree $d$. A network complexity with $d=20,25,30$ indicates on average, the initial nodes' outdegrees is 20,25,30. Peer's real behavior is represented by a pre-set normal distribution $\left(\mu_{r}=0.25,0.75, \sigma_{r}^{2}=0.2\right)$ rating score $r \in[0,1]$. As mentioned in Section 3, we assume the accurate direct trust ratings have already been generated. This is reasonable because any trust inference scheme must rely on an accurate trust rating scheme. It is meaningless to study the inference trust based on the direct trust rating if the direct trust rating is not reliable. So in our simulation, the direct trust rating $R \in[0,1]$ is generated with a normal distribution $\left(\mu_{R}=r, \sigma_{R}^{2}=0.1\right)$ based on peer's real behavior score $r$. The parameters in iteration function is set up as learning rate $\alpha=1$, discount factor $\gamma=1$ and adjusting factor $n_{a}=9$. To measure the performance under dynamic models, new transactions is continuously generated according to a poisson distribution with an arrival rate $\lambda=10$ to 50 transactions per service cycle, between a random source node and a random destination node. New node will randomly join the network, and peer leave/die also randomly happens. In such a dynamic model and in a real mobile ad hoc network, it is hard to achieve strictly convergence status. So the convergence in our simulation is $\epsilon$-convergence, and $\epsilon$-convergence is defined as that the variance between any peer's two consecutive trust tables is smaller than the pre-set threshold $\epsilon=0.02$.

\subsection{Results and Analysis}

Convergence Time. The convergence time is measured in terms of the number of time units needed to achieve $\epsilon$-convergence status. Figure 3 shows that cTrust only needs a small number of aggregation cycles before convergence. We also observe that convergence time increases as network complexity increases. As 


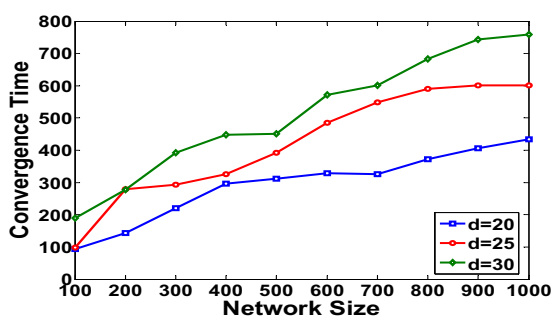

(a) Random Trust Topology

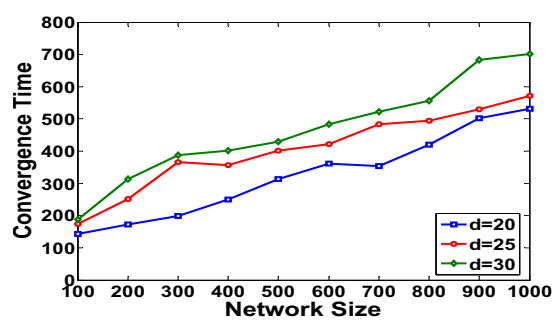

(b) Scale-Free Trust Topology

Fig. 3. Convergence Time

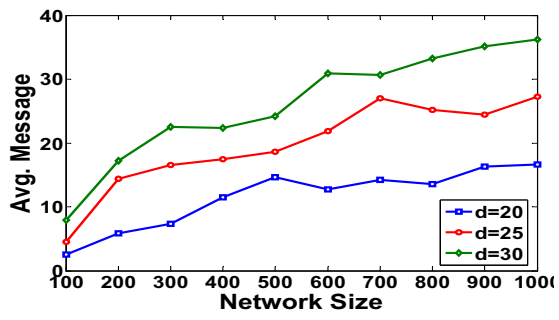

(a) Random Trust Topology

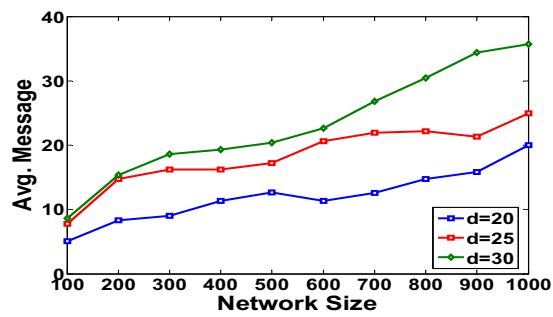

(b) Scale-Free Trust Topology

Fig. 4. Message Overhead

network size $N$ increases, convergence time increases relatively slowly $(O(n))$. This shows that cTrust features satisfactory scalability. We also observe that the trust topologies do not affect the convergence time so much as network size.

Communication Message Overhead. Figure 4 shows the average communication message overhead to achieve convergence per individual peer. cTrust greatly reduce the communication message overhead by using MDP model. This is because, in each iteration, each node only receive trust table for one of its most trusted neighbors (Equation (44)). The message overhead grows slowly as the network size grows, showing that cTrust is a lightweight scheme. In a network with high complexity, cTrust system incurs more message overheads. In a typical cTrust network, the average message overhead is affected by only network size $N$ and complexity $d$ and not affected by trust topology. As a result, the overhead curves for both topologies in the figures appear similar.

Average Trust Path Length. Figure 5 indicates the average length of a trust path starts from a source peer to a destination peer in convergence status. Generally, the trust path length increases with the network size and complexity, which indicates peers gain more remote trust information. In the scale-free trust topology, the trust path length is greatly reduced. This is because in scale-free trust topology most peers have only a few connections while some power peers control many links, making trust information hard to spread. In a complex network where trust information can be spread father, there are more longer trust paths and involve more trust transfers. 


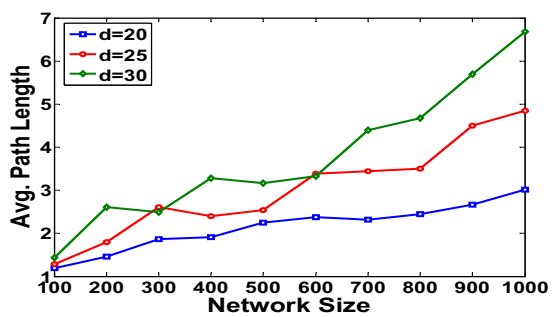

(a) Random Trust Topology

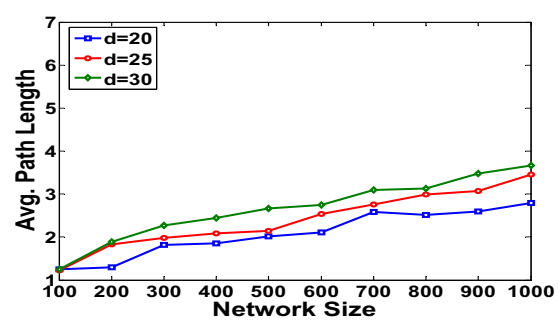

(b) Scale-Free Trust Topology

Fig. 5. Average Trust Path Length

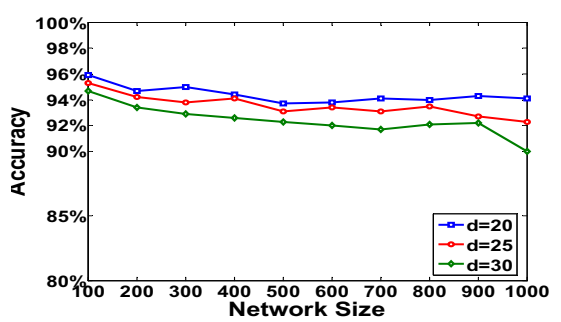

(a) Random Trust Topology

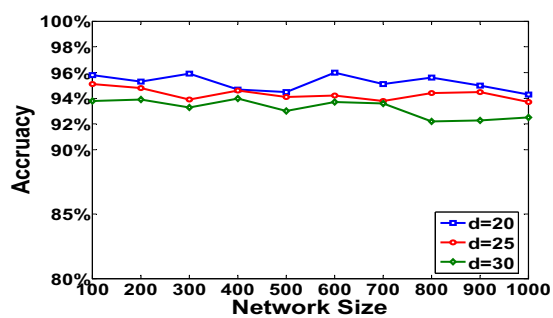

(b) Scale-Free Trust Topology

Fig. 6. Aggregation Accuracy

Accuracy. cTrust aggregation accuracy is measured by comparing all the inferred trust ratings with peers real behavior scores. The similarity is considered as aggregation accuracy. As shown in Figure 6, on average, cTrust aggregation accuracy is maintained above $90 \%$. The result is very encouraging because cTrust is a personalized trust system using inferred (not direct) trust and the information for each node to access is limited in CMANETs. As the network complexity increases, the accuracy decreases. This is because in complex networks, there are more long trust paths that involve more trust transfers, resulting in lower accuracy in inferred trust ratings due to multi-hop relationships. The accuracy in scale-free trust topology is slightly higher than in random trust topology. One reason is in scale-free trust topology, the average trust path is shorter which leads to high accuracy in trust transfer.

\section{Conclusion}

We have presented the cTrust scheme in CMANETs. cTrust scheme is aimed to provide a common framework to enable trust inferring in a CMANET trust landscape. We presented the trust transfer function, trust value iteration function, and the cTrust distribution trust aggregation algorithm. To validate our proposed algorithms and protocols, we conducted extensive evaluation based on NUS students trace data. The experimental results demonstrate that cTrust 
scheme trust aggregation is efficient. cTrust convergence time increases slowly with network size. Message overhead in cTrust is modest. The trust information spreads fast and extensively in CMANETs. The trust rating inference accuracy in cTrust scheme is over $90 \%$. We believe that cTrust establishes a solid foundation to design trust-enabled applications and middleware in CMANETs.

\section{References}

1. Buchegger, S., Boudec, J.Y.L.: A robust reputation system for mobile ad-hoc networks. Technical report, IC/2003/50, EPFL-IC-LCA (2003)

2. Buchegger, S., Boudee, J.Y.L.: Self-policing mobile ad hoc networks by reputation systems. IEEE Communications Magazine 43(7), 101-107 (2005)

3. Ganeriwal, S., Balzano, L.K., Srivastava, M.B.: Reputation-based framework for high integrity sensor networks. ACM Trans. Sen. Netw. 4(3), 1-37 (2008)

4. Kamvar, S.D., Schlosser, M.T., G.-Molina, H.: The eigentrust algorithm for reputation management in $\mathrm{p} 2 \mathrm{p}$ networks. In: Proceedings of the 12th International Conference on World Wide Web (WWW). pp. 640-651. Budapest,Hungary (May 20-24 2003)

5. Liu, C., Wu, J.: Routing in a cyclic mobispace. In: MobiHoc: Proceedings of the 9th ACM international symposium on Mobile ad hoc networking and computing, pp. 351-360 (2008)

6. Michiardi, P., Molva, R.: Core: A collaborative reputation mechanism to enforce node cooperation in mobile ad hoc networks. In: Sixth IFIP conference on security communications, and multimedia (CMS 2002), Portoroz, Slovenia (2002)

7. Srinivasan, V., Motani, M., Ooi, W.T.: Analysis and implications of student contact patterns derived from campus schedules. In: MobiCom 2006: Proceedings of the 12 th annual international conference on Mobile computing and networking, New York, NY, USA, pp. 86-97 (2006)

8. Srinivasan, V., Motani, M., Ooi, W.T.: CRAWDAD data set nus/contact, v. 200608-01 (August 2006), http://crawdad.cs.dartmouth.edu/nus/contact

9. Sun, Y.L., Yu, W., Han, Z., Liu, K.J.R.: Information theoretic framework of trust modeling and evaluation for ad hoc networks. IEEE Journal on Selected Areas in Communications 24(2) (2006)

10. Theodorakopoulos, G., Baras, J.: On trust models and trust evaluation metrics for ad hoc networks. IEEE Journal on Selected Areas in Communications 24(2) (February 2006)

11. Xiong, L., Liu, L.: Peertrust: supporting reputation-based trust for peer-to-peer electronic communities. IEEE Transactions on Knowledge and Data Engineering 16(7), 843-857 (2004)

12. Zhao, H., Li, X.: H-trust: A robust and lightweight group reputation system for peer-to-peer desktop grid. Journal of Computer Science and Technology (JCST) 24(5), 833-843 (2009)

13. Zhao, H., Li, X.: Vectortrust: The trust vector aggregation scheme for trust management in peer-to-peer networks. In: The 18th International Conference on Computer Communications and Networks (ICCCN 2009), San Francisco, CA USA, August 2-6 (2009)

14. Zhou, R., Hwang, K.: Powertrust: A robust and scalable reputation system for trusted peer-to-peer computing. IEEE Transactions on Parallel and Distributed Systems 18(4), 460-473 (2007) 